\title{
A Unified Family of Generalized $q$-Hermite Apostol Type Polynomials and its Applications
}

\author{
Subuhi Khan ${ }^{1 *}$ and Tabinda Nahid ${ }^{1}$
}

\begin{abstract}
The intended objective of this paper is to introduce a new class of generalized $q$-Hermite based Apostol type polynomials by combining the $q$-Hermite polynomials and a unified family of $q$-Apostol-type polynomials. The generating function, series definition and several explicit representations for these polynomials are established. The $q$-Hermite-Apostol Bernoulli, $q$-Hermite-Apostol Euler and $q$-Hermite-Apostol Genocchi polynomials are studied as special members of this family and corresponding relations for these polynomials are obtained.
\end{abstract}

Keywords: $q$-Hermite polynomials, Generalized $q$-Apostol type polynomials, Generalized $q$-Hermite Apostol type polynomials, Explicit representation

2010 AMS: 11B73, 11B83, 11B68

${ }^{1}$ Department of Mathematics, Aligarh Muslim University, Aligarh, India

*Corresponding author: subuhi2006@gmail.com

Received: 28 October 2018, Accepted: 12 November 2018, Available online: 22 March 2019

\section{Introduction and preliminaries}

The $q$-calculus has been extensively studied for a long time by many mathematicians, physicists and engineers. The $q$-calculus is a generalization of many subjects, like the hypergeometric series, complex analysis and particle physics. The $q$-analogues of many orthogonal polynomials and functions assume a very pleasant form reminding directly of their classical counterparts. The $q$-calculus is mostly being used by physicists at a high level. In short, $q$-calculus is quite a popular subject today.

Throughout the present paper, $\mathbb{C}$ indicates the set of complex numbers, $\mathbb{N}$ denotes the set of natural numbers and $\mathbb{N}_{0}$ indicates the set of non-negative integers. Further, the variable $q \in \mathbb{C}$ such that $|q|<1$. The following $q$-standard notations and definitions are taken from [1].

The $q$-analogue of the shifted factorial $(a)_{n}$ is defined by

$$
(a ; q)_{0}=1,(a ; q)_{n}=\prod_{m=0}^{n-1}\left(1-q^{m} a\right), n \in \mathbb{N} .
$$

The $q$-analogues of a complex number $a$ and of the factorial function are defined by

$$
\begin{aligned}
& {[a]_{q}=\frac{1-q^{a}}{1-q}, q \in \mathbb{C}-\{1\} ; \quad a \in \mathbb{C},} \\
& {[n]_{q} !=\prod_{m=1}^{n}[m]_{q}=\frac{(q ; q)_{n}}{(1-q)^{n}}, q \neq 1 ; n \in \mathbb{N},[0]_{q} !=1, q \in \mathbb{C} .}
\end{aligned}
$$


The $q$-binomial coefficient $\left[\begin{array}{l}n \\ k\end{array}\right]_{q}$ is defined by

$$
\left[\begin{array}{l}
n \\
k
\end{array}\right]_{q}=\frac{[n]_{q} !}{[k]_{q} ![n-k]_{q} !}=\frac{(q ; q)_{n}}{(q ; q)_{k}(q ; q)_{n-k}}, \quad k=0,1, \ldots, n .
$$

The $q$-exponential function is defined as:

$$
e_{q}(x)=\sum_{n=0}^{\infty} \frac{x^{n}}{[n]_{q} !}=\frac{1}{((1-q) x ; q)_{\infty}}, \quad|x|<|1-q|^{-1} .
$$

The $q$-Hermite polynomials are special or limiting cases of the orthogonal polynomials as they contain no parameter other than $q$ and appears to be at the bottom of a hierarchy of the classical $q$-orthogonal polynomials [2]. The $q$-Hermite polynomials constitute a 1-parameter family of orthogonal polynomials, which for $q=1$ reduce to the well known Hermite polynomials. We recall that the $q$-Hermite polynomials $H_{n, q}(x)$ is defined by the following generating function [3]:

$$
\begin{aligned}
& F_{q}(x, t):=F_{q}(t) e_{q}(x t)=\sum_{n=0}^{\infty} H_{n, q}(x) \frac{t^{n}}{[n]_{q} !}, \\
& F_{q}(t):=\sum_{n=0}^{\infty}(-1)^{n} q^{n(n-1) / 2} \frac{t^{2 n}}{[2 n]_{q} ! !}, \quad[2 n]_{q} ! !=[2 n]_{q}[2 n-2]_{q} \ldots .[2]_{q} . \\
& D_{q, x} H_{n, q}(x)=[n]_{q} H_{n-1, q}(x) .
\end{aligned}
$$

Recently, many mathematicians studied the unification of the Bernoulli and Euler polynomials. Luo and Srivastava $[4,5]$ introduced the generalized Apostol-Bernoulli polynomials $B_{n}^{(\alpha)}(x)$ of order $\alpha$. Further, the generalized Apostol-Euler polynomials $E_{n}^{(\alpha)}(x)$ of order $\alpha$ and the generalized Apostol-Genochhi polynomials $G_{n}^{(\alpha)}(x)$ of order $\alpha$ are investigated by Luo [6,7]. Thereafter, in 2014 Ernst [8] defined the $q$-analogues of the generalized Apostol type polynomials.

The generalized $q$-Apostol-Bernoulli polynomials $B_{n, q, \lambda}^{(\alpha)}(x)$ of order $\alpha \in \mathbb{N}_{0}$ are defined by the following generating function [8]:

$$
\left(\frac{t}{\lambda e_{q}(t)-1}\right)^{\alpha} e_{q}(x t)=\sum_{n=0}^{\infty} B_{n, q, \lambda}^{(\alpha)}(x) \frac{t^{n}}{[n]_{q} !} .
$$

The generalized $q$-Apostol-Euler polynomials $E_{n, q, \lambda}^{(\alpha)}(x)$ of order $\alpha \in \mathbb{N}_{0}$ are defined by the following generating function [8]:

$$
\left(\frac{2}{\lambda e_{q}(t)+1}\right)^{\alpha} e_{q}(x t)=\sum_{n=0}^{\infty} E_{n, q, \lambda}^{(\alpha)}(x) \frac{t^{n}}{[n]_{q} !}
$$

The generalized $q$-Apostol-Genocchi polynomials $G_{n, q, \lambda}^{(\alpha)}(x)$ of order $\alpha \in \mathbb{N}_{0}$ are defined by the following generating function [8]:

$$
\left(\frac{2 t}{\lambda e_{q}(t)+1}\right)^{\alpha} e_{q}(x t)=\sum_{n=0}^{\infty} G_{n, q, \lambda}^{(\alpha)}(x) \frac{t^{n}}{[n]_{q} !} .
$$

In view of equations (1.3)-(1.5), the generalized $q$-Apostol type polynomials $\mathscr{P}_{n, q, \beta}^{(\alpha)}(x ; k, a, b) \quad\left(\alpha \in \mathbb{N}_{0}, \lambda, a, b \in \mathbb{C}\right)$ of order $\alpha$ are defined by the following generating function:

$$
\left(\frac{2^{1-k} t^{k}}{\beta^{b} e_{q}(t)-a^{b}}\right)^{\alpha} e_{q}(x t)=\sum_{n=0}^{\infty} \mathscr{P}_{n, q, \beta}^{(\alpha)}(x ; k, a, b) \frac{t^{n}}{[n]_{q} !},
$$

where $\mathscr{P}_{n, q, \beta}^{(\alpha)}(k, a, b)=\mathscr{P}_{n, q, \beta}^{(\alpha)}(0 ; k, a, b)$ are the $q$-Apostol type numbers of order $\alpha$.

If we take the limit $q \rightarrow 1$, the generalized $q$-Apostol type polynomials defined by equation (1.6) reduces to the unified Apostol type polynomials [9]. In fact, the following special cases hold:

$$
\lim _{q \rightarrow 1} \mathscr{P}_{n, q, \lambda}^{(\alpha)}(x ; 1,1,1)=B_{n, \lambda}^{(\alpha)}(x),
$$




$$
\begin{aligned}
& \lim _{q \rightarrow 1} \mathscr{P}_{n, q, \lambda}^{(\alpha)}(x ; 0,-1,1)=E_{n, \lambda}^{(\alpha)}(x), \\
& \lim _{q \rightarrow 1} \mathscr{P}_{n, q, \frac{\lambda}{2}}^{(\alpha)}(x ; 1,-1 / 2,1)=G_{n, \lambda}^{(\alpha)}(x),
\end{aligned}
$$

where $B_{n, \lambda}^{(\alpha)}(x), E_{n, \lambda}^{(\alpha)}(x)$ and $G_{n, \lambda}^{(\alpha)}(x)$ are the generalized forms of the Apostol-Bernoulli, Apostol-Euler and Apostol-Genocchi polynomials.

In the current article, the $q$-Hermite-Apostol type polynomials are introduced and their explicit relations are proved. The corresponding results for the $q$-Hermite-Apostol Bernoulli, $q$-Hermite-Apostol Euler and $q$-Hermite-Apostol Genocchi polynomials are established.

\section{Generalized $q$-Hermite Apostol type polynomials}

In this section, a new hybrid class of the generalized $q$-Hermite-Apostol type polynomials $(\operatorname{GqHATyP})$, denoted by ${ }_{H} \mathscr{P}_{n, q, \beta}^{(\alpha)}(x ; k, a, b)$ is introduced by convoluting the $q$-Hermite polynomials and generalized $q$-Apostol type polynomials. In order to establish the generating function for the these polynomials, the following result is proved:

Theorem 2.1. The following generating function for the generalized $q$-Hermite based Apostol type polynomials ${ }_{H} \mathscr{P}_{n, q, \beta}^{(\alpha)}(x ; k, a, b)(\alpha \in$ $\left.\mathbb{N}_{0}, \lambda, a, b \in \mathbb{C}\right)$ holds true:

$$
\left(\frac{2^{1-k} t^{k}}{\beta^{b} e_{q}(t)-a^{b}}\right)^{\alpha} F_{q}(t) e_{q}(x t)=\sum_{n=0}^{\infty} H_{n, q, \beta} \mathscr{P}^{(\alpha)}(x ; k, a, b) \frac{t^{n}}{[n]_{q} !},
$$

Proof. Expanding the exponential function $e_{q}(x t)$ and then replacing the powers of $x$, i.e. $x^{0} ; x^{1} ; x^{2} ; \cdots ; x^{n}$ by the correlating q-Hermite polynomials $H_{0, q}(x) ; H_{1, q}(x) ; \cdots ; H_{n, q}(x)$ in the 1.h.s. of equation (1.6) and after summing up the terms of the resultant equation and denoting the resultant GqHATyP in the r.h.s. by ${ }_{H} \mathscr{P}_{n, q, \beta}^{(\alpha)}(x ; k, a, b)$, assertion (2.1) is proved.

Taking $x=0$ in equation (2.1), we get

$$
{ }_{H} \mathscr{P}_{n, q, \beta}^{(\alpha)}(k, a, b)={ }_{H} \mathscr{P}_{n, q, \beta}^{(\alpha)}(0 ; k, a, b),
$$

where ${ }_{H} \mathscr{P}_{n, q, \beta}^{(\alpha)}(k, a, b)$ are the $q$-Hermite Apostol type numbers of order $\alpha$.

Next, the series expansions for the GqHATyP ${ }_{H} \mathscr{P}_{n, q, \beta}^{(\alpha)}(x ; k, a, b)$ is obtained by proving the following result:

Theorem 2.2. The following series expansions for the generalized $q$-Hermite based Apostol type polynomials ${ }_{H} \mathscr{P}_{n, q, \beta}^{(\alpha)}(x ; k, a, b)$ hold true:

${ }_{H} \mathscr{P}_{n, q, \beta}^{(\alpha)}(x ; k, a, b)=\sum_{r=0}^{n}\left[\begin{array}{l}n \\ r\end{array}\right]_{q} \mathscr{P}_{r, q, \beta}^{(\alpha)}(k, a, b) H_{n-r, q}(x)$,

${ }_{H} \mathscr{P}_{n, q, \beta}^{(\alpha)}(x ; k, a, b)=\sum_{r=0}^{n}\left[\begin{array}{l}n \\ r\end{array}\right]_{q}{ }_{H} \mathscr{P}_{r, q, \beta}^{(\alpha)}(k, a, b) x^{n-r}$.

Proof. Utilizing equations (1.2) and (1.6) in the 1.h.s. of generating function (2.1) and then using Cauchy-product rule in the 1.h.s. of the resultant equation, it follows that

$$
\sum_{n=0}^{\infty} \sum_{r=0}^{n}\left[\begin{array}{l}
n \\
r
\end{array}\right]_{q} \mathscr{P}_{r, q, \beta}^{(\alpha)}(k, a, b) H_{n-r, q}(x) \frac{t^{n}}{[n]_{q} !}=\sum_{n=0}^{\infty} H_{n, q, \beta}^{(\alpha)}(x ; k, a, b) \frac{t^{n}}{[n]_{q} !} .
$$

Equating the coefficients of identical powers of $t$ in both sides of equation (2.4), assertion (2.2) follows.

Utilizing equation (1.1) in the 1.h.s. of generating function (2.1), it follows that

$$
\sum_{n=0}^{\infty} H_{n, q, \beta} \mathscr{P}_{n, k}^{(\alpha)}(x ; k, a, b) \frac{t^{n}}{[n]_{q} !}=\sum_{n=0}^{\infty} x^{n} \frac{t^{n}}{[n]_{q} !} \sum_{r=0}^{\infty} H_{r, q, \beta} \mathscr{P}_{r, \beta}^{(\alpha)}(k, a, b) \frac{t^{r}}{[n]_{r} !},
$$

which on applying the Cauchy product rule in the r.h.s. and then comparing the coefficients of same powers of $t$ in both sides of resultant equation yields assertion (2.3). 
S. No. $k ; a ; b ; \beta$ Generating function

Name of the polynomials

I. $\quad k=1 ; a=1 ; \quad\left(\frac{t}{\lambda e_{q}(t)-1}\right)^{(\alpha)} F_{q}(t) e_{q}(x t)=\sum_{n=0}^{\infty} H_{H, q, \lambda} B_{n)}^{(\alpha)}(x) \frac{t^{n}}{[n]_{q} !}$

The generalized $q$-Hermite-

$b=1 ; \beta=\lambda$

Apostol Bernoulli polynomials (GqHABP)

II. $\quad k=0 ; \quad a=-1 ; \quad\left(\frac{2}{\lambda e_{q}(t)+1}\right)^{(\alpha)} F_{q}(t) e_{q}(x t)=\sum_{n=0}^{\infty}{ }_{H} E_{n, q, \lambda}^{(\alpha)}(x) \frac{t^{n}}{[n]_{q} !}$

The generalized $q$-Hermite-

$b=1 ; \beta=\lambda$

Apostol Euler polynomials (GqHAEP)

III. $\quad k=1 ; a=-1 / 2 ; \quad\left(\frac{2 t}{\lambda e_{q}(t)+1}\right)^{(\alpha)} F_{q}(t) e_{q}(x t)=\sum_{n=0}^{\infty}{ }_{H} G_{n, q, \lambda}^{(\alpha)}(x) \frac{t^{n}}{[n]_{q} !} \quad$ The generalized $q$-Hermite$b=1 ; \beta=\lambda / 2$

Apostol Genocchi polynomials (GqHAGP)

Table 1. Certain members belonging to the generalized $q$-Hermite-Apostol family

Different members of the generalized $q$-Hermite-Apostol family can be obtained by making suitable selections of the parameters $k, a, b$ and $\beta$ in generating relation (2.1). Some of these members are listed in Table 1.

Proposition 2.3. The following relations for the generalized $q$-Hermite based Apostol type polynomials ${ }_{H} \mathscr{P}_{n, q, \beta}^{(\alpha)}(x ; k, a, b)$ holds true:

$$
\begin{aligned}
D_{q, t} e_{q}(x t) & =x e_{q}(x t) \\
D_{q, x}\left({ }_{H} \mathscr{P}_{n, q, \beta}^{(\alpha)}(x ; k, a, b)\right) & =[n]_{q H} \mathscr{P}_{n-1, q, \beta}^{(\alpha)}(x ; k, a, b) .
\end{aligned}
$$

Theorem 2.4. For each $n \in \mathbb{N}$ and for the q-commuting variables $x$ and $u$ such that $x u=q u x$, the generalized $q$-Hermite based Apostol type polynomials ${ }_{H} \mathscr{P}_{k, q, \beta}^{(\alpha)}(x ; k, a, b)$ satisfy the following relations:

$$
\begin{array}{r}
\mathscr{P}_{n, q, \beta}^{(\alpha+\gamma)}(x ; k, a, b)=\sum_{r=0}^{n}\left[\begin{array}{l}
n \\
r
\end{array}\right]_{q} \mathscr{P}_{r, q, \beta}^{(\alpha)}(x ; k, a, b) \mathscr{P}_{n-r, q, \beta}^{(\gamma)}(k, a, b) . \\
\mathscr{P}_{n, q, \beta}^{(\alpha+\gamma)}(x+u ; k, a, b)=\sum_{r=0}^{n}\left[\begin{array}{c}
n \\
r
\end{array}\right]_{q} \mathscr{P}_{r, q, \beta}^{(\alpha)}(x ; k, a, b) \mathscr{P}_{n-r, q, \beta}^{(\gamma)}(u ; k, a, b) .
\end{array}
$$

Proof. Replacing $\alpha$ by $\alpha+\gamma$ in definition (2.1), we have

$$
\begin{aligned}
\sum_{n=0}^{\infty} H_{n, q, \beta} \mathscr{P}_{n+\gamma)}^{(\alpha+\gamma, a, b) \frac{t^{n}}{[n]_{q} !}} & =\left(\frac{2^{1-k} t^{k}}{\beta^{b} e_{q}(t)-a^{b}}\right)^{\alpha+\gamma} F_{q}(t) e_{q}(x t) \\
& =\left(\sum_{r=0}^{\infty} H_{r, q, \beta} \mathscr{P}_{r, k}^{(\alpha)}(x ; k, b) \frac{t^{r}}{[r]_{q} !}\right)\left(\sum_{n=0}^{\infty} \mathscr{P}_{n, q, \beta}^{(\gamma)}(k, a, b) \frac{t^{n}}{[n]_{q} !}\right) .
\end{aligned}
$$

Using Cauchy-product rule in the r.h.s. of above equation, it follows that

$$
\sum_{n=0}^{\infty} H_{n, q, \beta} \mathscr{P}_{n+\gamma}^{(\alpha+\gamma)}(x ; k, a, b) \frac{t^{n}}{[n]_{q} !}=\sum_{n=0}^{\infty} \sum_{r=0}^{n}\left[\begin{array}{l}
n \\
r
\end{array}\right]_{q}{ }_{H_{r, q, \beta}} \mathscr{P}_{r, \alpha, a, b)}^{(\alpha)}\left(x ; \mathscr{P}_{n-r, q, \beta}^{(\gamma)}(k, a, b) \frac{t^{n}}{[n]_{q} !}\right.
$$

Equating the coefficients of identical powers of $t$ in both sides of equation (2.7), assertion (2.5) follows. Further, replacing $\alpha$ by $\alpha+\gamma$ and $x$ by $x+u$ in Definition 2.1 and proceeding on the same lines of proof as above, assertion (2.6) follows.

Theorem 2.5. For each $n \in \mathbb{N}$ and for the q-commuting variables $x$ and $u$ such that $x u=q u x$, the generalized $q$-Hermite based Apostol type polynomials ${ }_{H} \mathscr{P}_{k, q, \beta}^{(\alpha)}(x ; k, a, b)$ satisfy the following relation:

$$
\beta_{H}^{b} \mathscr{P}_{n, q, \beta}^{(\alpha)}(x+1 ; k, a, b)-a^{b}{ }_{H} \mathscr{P}_{n, q, \beta}^{(\alpha)}(x ; k, a, b)=\frac{2^{1-k}[n]_{q} !}{[n-k]_{q} !} H_{n-k, q, \beta} \mathscr{P}_{n, k, a, b)}^{(\alpha-1)}(x)
$$




\section{S. No. Special polynomials Results}

I. GqHABP $\quad{ }_{H} B_{n, q, \lambda}(\alpha)(x)=\sum_{r=0}^{n}\left[\begin{array}{l}n \\ r\end{array}\right]_{q} H_{r, q, \lambda} B_{r}^{(\alpha)} x^{n-r}$

${ }_{H} B_{n, q, \lambda}^{(\alpha)}(x)$

${ }_{H} B_{n, q, \lambda}^{(\alpha+\gamma)}(x)=\sum_{r=0}^{n}\left[\begin{array}{l}n \\ r\end{array}\right]_{q}{ }_{H} B_{r, q, \lambda}^{(\alpha)}(x) B_{n-r, q, \lambda}^{(\gamma)}$

${ }_{H} B_{n, q, \lambda}^{(\alpha+\gamma)}(x+u)=\sum_{r=0}^{n}\left[\begin{array}{l}n \\ r\end{array}\right]_{q}{ }_{H} B_{r, q, \lambda}^{(\alpha)}(x) B_{n-r, q, \lambda}^{(\gamma)}(u)$

$\beta^{b}{ }_{H} B_{n, q, \lambda}^{(\alpha)}(x+1)-a^{b}{ }_{H} B_{n, q, \lambda}^{(\alpha)}(x)=\frac{2^{1-k}[n] q !}{[n-k]_{q} !}{ }_{H} B_{n-k, q, \lambda}^{(\alpha-1)}(x)$

$$
{ }_{H} E_{n, q, \lambda}^{(\alpha)}(x)=\sum_{r=0}^{n}\left[\begin{array}{l}
n \\
r
\end{array}\right]_{q} E_{r, q, \lambda}^{(\alpha)} H_{n-r, q}(x)
$$

II. GqHAEP

$$
{ }_{H} E_{n, q, \lambda}(\alpha)(x)=\sum_{r=0}^{n}\left[\begin{array}{l}
n \\
r
\end{array}\right]_{q}{ }_{H} E_{r, q, \lambda}^{(\alpha)} x^{n-r}
$$

${ }_{H} E_{n, q, \lambda}^{(\alpha)}(x)$

${ }_{H} E_{n, q, \lambda}^{(\alpha+\gamma)}(x)=\sum_{r=0}^{n}\left[\begin{array}{l}n \\ r\end{array}\right]_{q}{ }_{H} E_{r, q, \lambda}^{(\alpha)}(x) E_{n-r, q, \lambda}^{(\gamma)}$

${ }_{H} E_{n, q, \lambda}^{(\alpha+\gamma)}(x+u)=\sum_{r=0}^{n}\left[\begin{array}{l}n \\ r\end{array}\right]_{q}{ }_{H} E_{r, q, \lambda}^{(\alpha)}(x) E_{n-r, q, \lambda}^{(\gamma)}(u)$

$\beta^{b}{ }_{H} E_{n, q, \lambda}^{(\alpha)}(x+1)-a_{H}^{b} E_{n, q, \lambda}^{(\alpha)}(x)=\frac{2^{1-k}[n]_{q} !}{[n-k]_{q} !}{ }_{H} E_{n-k, q, \lambda}^{(\alpha-1)}(x)$

$$
{ }_{H} G_{n, q, \lambda}^{(\alpha)}(x)=\sum_{r=0}^{n}\left[{ }^{n}{ }_{r}^{n}\right]_{q} G_{r, q, \lambda}^{(\alpha)} H_{n-r, q}(x)
$$

II. GqHAGP

$$
\begin{aligned}
& { }_{H} G_{n, q, \lambda}(\alpha)(x)=\sum_{r=0}^{n}\left[\begin{array}{l}
n \\
r
\end{array}\right]_{q}{ }_{H} G_{r, q, \lambda}^{(\alpha)} x^{n-r} \\
& { }_{H} G_{n, q, \lambda}^{(\alpha+\gamma)}(x)=\sum_{r=0}^{n}\left[\begin{array}{c}
n \\
r
\end{array}\right]_{q}{ }_{H} G_{r, q, \lambda}^{(\alpha)}(x) G_{n-r, q, \lambda}^{(\gamma)} \\
& { }_{H} G_{n, q, \lambda}^{(\alpha+\gamma)}(x+u)=\sum_{r=0}^{n}\left[\begin{array}{l}
n \\
r
\end{array}\right]_{q}{ }_{H} G_{r, q, \lambda}^{(\alpha)}(x) G_{n-r, q, \lambda}^{(\gamma)}(u) \\
& \beta^{b}{ }_{H} G_{n, q, \lambda}^{(\alpha)}(x+1)-a^{b}{ }_{H} G_{n, q, \lambda}^{(\alpha)}(x)=\frac{2^{1-k}[n]_{q} !}{[n-k]_{q} !}{ }_{H} G_{n-k, q, \lambda}^{(\alpha-1)}(x)
\end{aligned}
$$$$
{ }_{H} G_{n, q, \lambda}^{(\alpha)}(x)
$$

Table 2. Certain results for the $\operatorname{GqHABP}_{H} B_{n, q, \lambda}^{(\alpha)}(x), \operatorname{GqHAEP}_{H} E_{n, q, \lambda}^{(\alpha)}(x)$ and $\operatorname{GqHAGP}_{H} G_{n, q, \lambda}^{(\alpha)}(x)$

Proof. From generating relation (2.1), we have

$$
\begin{gathered}
\sum_{n=0}^{\infty} \beta_{H}^{b} \mathscr{P}_{n, q, \beta}^{(\alpha)}(x+1 ; k, a, b) \frac{t^{n}}{[n]_{q} !}-\sum_{n=0}^{\infty} a_{H}^{b} \mathscr{P}_{n, q, \beta}^{(\alpha)}(x ; k, a, b) \frac{t^{n}}{[n]_{q} !} \\
=\beta^{b}\left(\frac{2^{1-k} t^{k}}{\beta^{b} e_{q}(t)-a^{b}}\right)^{\alpha} F_{q}(t) e_{q}((x+1) t)-a^{b}\left(\frac{2^{1-k} t^{k}}{\beta^{b} e_{q}(t)-a^{b}}\right)^{\alpha} F_{q}(t) e_{q}(x t) \\
=\left(\frac{2^{1-k} t^{k}}{\beta^{b} e_{q}(t)-a^{b}}\right)^{\alpha} F_{q}(t) e_{q}(x t)\left(\beta^{b} e_{q}(t)-a^{b}\right) \\
\sum_{n=0}^{\infty}\left(\beta^{b}{ }_{H} \mathscr{P}_{n, q, \beta}^{(\alpha)}(x+1 ; k, a, b)-a_{H}^{b} \mathscr{P}_{n, q, \beta}^{(\alpha)}(x ; k, a, b)\right) \frac{t^{n}}{[n]_{q} !}=\sum_{n=0}^{\infty} 2^{1-k} \mathscr{P}_{n, q, \beta}^{(\alpha-1)}(x ; k, a, b) \frac{t^{n+k}}{[n]_{q} !} .
\end{gathered}
$$

Equating the coefficients of same powers of $t$ in both sides of the above equation, assertion (2.8) follows.

In view of Table 1 , ceratin results for the $\operatorname{GqHABP}_{H} B_{n, q, \lambda}^{(\alpha)}(x), \operatorname{GqHAEP}_{H} E_{n, q, \lambda}^{(\alpha)}(x)$ and $\operatorname{GqHAGP}_{H} G_{n, q, \lambda}^{(\alpha)}(x)$ are established and are given in Table 2.

In the next section, certain explicit representations for the $\operatorname{GqHATyP}_{H} \mathscr{P}_{n, q, \beta}^{(\alpha)}(x ; k, a, b)$ are established. 


\section{Explicit representations}

In order to derive the explicit representations for the $\operatorname{GqHATyP}_{H} \mathscr{P}_{n, q, \beta}^{(\alpha)}(x ; k, a, b)$, we recall the following definition:

Definition 3.1. The generalized $q$-Stirling numbers $S_{q}(n, v, a, b, \beta)$ of the second kind of order $v$ is defined as [10]:

$$
\sum_{n=0}^{\infty} S_{q}(n, v, a, b, \beta) \frac{t^{n}}{[n]_{q} !}=\frac{\left(\beta^{b} e_{q}(t)-a^{b}\right)^{v}}{[v]_{q} !} .
$$

Theorem 3.2. The following explicit formula for the generalized $q$-Hermite based Apostol type polynomials ${ }_{H} \mathscr{P}_{n, q, \beta}^{(\alpha)}(x ; k, a, b)$ in terms of the generalized $q$-Stirling numbers of the second kind $S_{q}(n, v, a, b, \beta)$ holds true:

$$
{ }_{H} \mathscr{P}_{n-v k, q, \beta}^{(\alpha)}(x ; k, a, b)=2^{v(k-1)} \frac{[v]_{q} ![n-v k]_{q} !}{[n]_{q} !} \sum_{l=0}^{n}\left[\begin{array}{l}
n \\
l
\end{array}\right]_{q}{ }_{q} \mathscr{P}_{l, q, \beta}^{(v-\alpha)}(x ; k, a, b) S_{q}(n-l, v, a, b, \beta) .
$$

Proof. From generating relation (2.1), we have

$$
\begin{aligned}
\sum_{n=0}^{\infty}{ }_{H} \mathscr{P}_{n, q, \beta}^{(\alpha)}(x ; k, a, b) \frac{t^{n}}{[n]_{q} !} & =\left(\frac{2^{1-k} t^{k}}{\beta^{b} e_{q}(t)-a^{b}}\right)^{\alpha} F_{q}(t) e_{q}(x t) \frac{\left(\beta^{b} e_{q}(t)-a^{b}\right)^{v}}{[v]_{q} !}\left(\frac{[v]_{q} !}{\left(\beta^{b} e_{q}(t)-a^{b}\right)^{v}}\right) \\
& =\frac{[v]_{q} !}{\left(2^{1-k} t^{k}\right)^{v}} \sum_{l=0}^{\infty} H_{H} \mathscr{P}_{l, q, \beta}^{(\alpha-v)}(x ; k, a, b) \frac{t^{l}}{[l]_{q} !}\left(\sum_{n=0}^{\infty} S_{q}(n, v, a, b, \beta) \frac{t^{n}}{[n]_{q} !}\right) .
\end{aligned}
$$

Applying the Cauchy-product rule on the r.h.s. of the above equation, it follows that

$$
\begin{aligned}
\sum_{n=0}^{\infty}{ }_{H} \mathscr{P}_{n, q, \beta}^{(\alpha)}(x ; k, a, b) \frac{t^{n+v k}}{[n]_{q} !}= & {[v]_{q} ! 2^{(k-1) v} \sum_{n=0}^{\infty}\left\{\sum_{l=0}^{n}\left[\begin{array}{c}
n \\
l
\end{array}\right]_{q}{ }_{H} \mathscr{P}_{l, q, \beta}^{(\alpha-v)}(x ; k, a, b)\right.} \\
& \left.\times S_{q}(n-l, v, a, b, \beta)\right\} \frac{t^{n}}{[n]_{q} !} .
\end{aligned}
$$

Equating the coefficients of identical powers of $t$ in both sides of equation (3.2) yields assertion (3.1).

Theorem 3.3. The following explicit relation for the generalized $q$-Hermite based Apostol type polynomials ${ }_{H} \mathscr{P}_{n, q, \beta}^{(\alpha)}(x ; k, a, b)$ in terms of the generalized $q$-Apostol Bernoulli polynomials $B_{n, q, \lambda}(x)$ holds true:

$$
\begin{aligned}
H_{n, q, \beta} \mathscr{P}^{(\alpha)}(x ; k, a, b)= & \frac{1}{[n+1]_{q}}\left\{\lambda \sum_{r=0}^{n+1}\left[\begin{array}{c}
n+1 \\
r
\end{array}\right]_{q} \sum_{m=0}^{r}\left[\begin{array}{c}
r \\
m
\end{array}\right]_{q} B_{n+1-r, q, \lambda}(x)\right. \\
& \left.-\sum_{m=0}^{n+1}\left[\begin{array}{c}
n+1 \\
m
\end{array}\right]_{q} B_{n+1-m, q, \lambda}(x)\right\}_{H} \mathscr{P}_{m, q, \beta}^{(\alpha)}(k, a, b) .
\end{aligned}
$$

Proof. Consider generating function (2.1) in the following form:

$$
\left(\frac{2^{1-k} t^{k}}{\beta^{b} e_{q}(t)-a^{b}}\right)^{\alpha} F_{q}(t) e_{q}(x t)=\left(\frac{2^{1-k} t^{k}}{\beta^{b} e_{q}(t)-a^{b}}\right)^{\alpha} F_{q}(t)\left(\frac{t}{\lambda e_{q}(t)-1}\right) \frac{\lambda e_{q}(t)-1}{t} e_{q}(x t),
$$

which on simplifying and rearranging the terms becomes

$$
\begin{aligned}
\left(\frac{2^{1-k} t^{k}}{\beta^{b} e_{q}(t)-a^{b}}\right)^{\alpha} F_{q}(t) e_{q}(x t) & =\left(\frac{2^{1-k} t^{k}}{\beta^{b} e_{q}(t)-a^{b}}\right)^{\alpha} F_{q}(t)\left(\frac{t}{\lambda e_{q}(t)-1} e_{q}(x t)\right) \frac{\lambda}{t} e_{q}(t) \\
& -\frac{1}{t}\left(\frac{2^{1-k} t^{k}}{\beta^{b} e_{q}(t)-a^{b}}\right)^{\alpha} F_{q}(t)\left(\frac{t}{\lambda e_{q}(t)-1} e_{q}(x t)\right) .
\end{aligned}
$$

Using equations (1.3) and (2.1) in equation (3.4), we have

$$
\begin{aligned}
\sum_{n=0}^{\infty}{ }_{H} \mathscr{P}_{n, q, \beta}^{(\alpha)}(x ; k, a, b) \frac{t^{n}}{[n]_{q} !} & =\frac{1}{t}\left(\lambda \sum_{m=0}^{\infty}{ }_{H} \mathscr{P}_{m, q, \beta}^{(\alpha)}(k, a, b) \frac{t^{m}}{[m]_{q} !} \sum_{n=0}^{\infty} B_{n, q}(x ; \lambda) \frac{t^{n}}{[n]_{q} !} \sum_{r=0}^{\infty} \frac{t^{r}}{[r]_{q} !}\right. \\
& \left.-\sum_{m=0}^{\infty} H_{m, q, \beta}^{(\alpha)}(k, a, b) \frac{t^{m}}{[m]_{q} !} \sum_{n=0}^{\infty} B_{n, q}(x ; \lambda) \frac{t^{n}}{[n]_{q} !}\right) .
\end{aligned}
$$


S. No. Special polynomials

\section{Explicit representations}

$$
{ }_{H} B_{n-v, q, \lambda}^{(\alpha)}(x)=\frac{[v]_{q} ![n-v]_{q} !}{[n]_{q} !} \sum_{l=0}^{n}\left[\begin{array}{l}
n \\
l
\end{array}\right]_{q}{ }_{H} B_{l, q, \lambda}^{v-\alpha}(x) S(n-l, v, 1,1, \lambda)
$$

I. GqHABP ${ }_{H} B_{n, q, \lambda}^{(\alpha)}(x)=\frac{1}{[n+1]_{q}}\left\{\lambda \sum_{r=0}^{n+1}\left[\begin{array}{c}n+1 \\ r\end{array}\right]_{q} \sum_{m=0}^{r}\left[\begin{array}{c}r \\ m\end{array}\right]_{q} B_{n+1-r, q, \lambda}(x)-\sum_{m=0}^{n+1}\left[\begin{array}{c}n+1 \\ m\end{array}\right]_{q} B_{n+1-m, q, \lambda}(x)\right\} H B_{m, q, \lambda}^{(\alpha)}$

$$
\begin{aligned}
{ }_{H} B_{n, q, \lambda}^{(\alpha)}(x) \quad{ }_{H} B_{n, q, \lambda}^{(\alpha)}(x) & =\frac{1}{2} \sum_{m=0}^{n}\left\{\lambda \sum_{r=0}^{n}\left[\begin{array}{c}
n \\
r
\end{array}\right]_{q} E_{n-r, q, \lambda}(x)+E_{n-m, q, \lambda}(x)\right\}{ }_{H} B_{m, q, \lambda}^{(\alpha)} \\
{ }_{H} B_{n, q, \lambda}^{(\alpha)}(x) & =\frac{1}{2[n+1]_{q}}\left\{\lambda \sum_{r=0}^{n+1}\left[\begin{array}{c}
n+1 \\
r
\end{array}\right]_{q} \sum_{m=0}^{r}\left[\begin{array}{c}
r \\
m
\end{array}\right]_{q} G_{n+1-r, q, \lambda}(x)-\sum_{m=0}^{n+1}\left[\begin{array}{c}
n+1 \\
m
\end{array}\right]_{q} G_{n+1-m, q, \lambda}(x)\right\}{ }_{H} B_{m, q, \lambda}^{(\alpha)}
\end{aligned}
$$

$$
{ }_{H} E_{n, q, \lambda}^{(\alpha)}(x)=\frac{[v]_{q} !}{2^{v}} \sum_{l=0}^{n}\left[\begin{array}{l}
n \\
l
\end{array}\right]_{q} H E_{l, q, \lambda}^{v-\alpha}(x) S(n-l, v,-1,1, \lambda)
$$

II. GqHAEP ${ }_{H} E_{n, q, \lambda}^{(\alpha)}(x)=\frac{1}{[n+1]_{q}}\left\{\lambda \sum_{r=0}^{n+1}\left[\begin{array}{c}n+1 \\ r\end{array}\right]_{q} \sum_{m=0}^{r}\left[\begin{array}{c}r \\ m\end{array}\right]_{q} B_{n+1-r, q, \lambda}(x)-\sum_{m=0}^{n+1}\left[\begin{array}{c}n+1 \\ m\end{array}\right]_{q} B_{n+1-m, q, \lambda}(x)\right\} H E_{m, q, \lambda}^{(\alpha)}$

$$
{ }_{H} E_{n, q, \lambda}^{(\alpha)}(x) \quad{ }_{H} E_{n, q, \lambda}^{(\alpha)}(x)=\frac{1}{2} \sum_{m=0}^{n}\left\{\lambda \sum_{r=0}^{n}\left[\begin{array}{l}
n \\
r
\end{array}\right]_{q} E_{n-r, q, \lambda}(x)+E_{n-m, q, \lambda}(x)\right\}{ }_{H} E_{m, q, \lambda}^{(\alpha)}
$$$$
{ }_{H} E_{n, q, \lambda}^{(\alpha)}(x)=\frac{1}{2[n+1]_{q}}\left\{\lambda \sum_{r=0}^{n+1}\left[\begin{array}{c}
n+1 \\
r
\end{array}\right]_{q} \sum_{m=0}^{r}\left[\begin{array}{c}
r \\
m
\end{array}\right]_{q} G_{n+1-r, q, \lambda}(x)-\sum_{m=0}^{n+1}\left[\begin{array}{c}
n+1 \\
m
\end{array}\right]_{q} G_{n+1-m, q, \lambda}(x)\right\} H E_{m, q, \lambda}^{(\alpha)}
$$

$$
{ }_{H} G_{n-v, q, \lambda}^{(\alpha)}(x)=\frac{[v]_{q} ![n-v]_{q} !}{[n]_{q} !} \sum_{l=0}^{n}\left[\begin{array}{l}
n \\
l
\end{array}\right]_{q}{ }_{H} G_{l, q, \lambda}^{v-\alpha}(x) S(n-l, v,-1 / 2,1, \lambda / 2)
$$

III. GqHAGP ${ }_{H} G_{n, q, \lambda}^{(\alpha)}(x)=\frac{1}{[n+1]_{q}}\left\{\lambda \sum_{r=0}^{n+1}\left[\begin{array}{c}n+1 \\ r\end{array}\right]_{q} \sum_{m=0}^{r}\left[\begin{array}{c}r \\ m\end{array}\right]_{q} B_{n+1-r, q, \lambda}(x)-\sum_{m=0}^{n+1}\left[\begin{array}{c}n+1 \\ m\end{array}\right]_{q} B_{n+1-m, q, \lambda}(x)\right\}{ }_{H} G_{m, q, \lambda}^{(\alpha)}$

$$
\begin{aligned}
{ }_{H} G_{n, q, \lambda}^{(\alpha)}(x) \quad{ }_{H} G_{n, q, \lambda}^{(\alpha)}(x) & =\frac{1}{2} \sum_{m=0}^{n}\left\{\lambda \sum_{r=0}^{n}\left[\begin{array}{l}
n \\
r
\end{array}\right]_{q} E_{n-r, q, \lambda}(x)+E_{n-m, q, \lambda}(x)\right\}{ }_{H} G_{m, q, \lambda}^{(\alpha)} \\
{ }_{H} G_{n, q, \lambda}^{(\alpha)}(x) & =\frac{1}{2[n+1]_{q}}\left\{\lambda \sum_{r=0}^{n+1}\left[\begin{array}{c}
n+1 \\
r
\end{array}\right]_{q} \sum_{m=0}^{r}\left[\begin{array}{c}
r \\
m
\end{array}\right]_{q} G_{n+1-r, q, \lambda}(x)-\sum_{m=0}^{n+1}\left[\begin{array}{c}
n+1 \\
m
\end{array}\right]_{q} G_{n+1-m, q, \lambda}(x)\right\}{ }_{H} G_{m, q, \lambda}^{(\alpha)}
\end{aligned}
$$

Table 3. Explicit representations for the $\operatorname{GqHABP}_{H} B_{n, q, \lambda}^{(\alpha)}(x), \operatorname{GqHAEP}_{H} E_{n, q, \lambda}^{(\alpha)}(x)$ and $\operatorname{GqHAGP}_{H} G_{n, q, \lambda}^{(\alpha)}(x)$

Comparing the coefficients of identical powers of $t$ in both sides of equation (3.5) yields assertion (3.3).

Similarly, we can prove the following results:

Corollary 3.4. The following explicit relation for the generalized $q$-Hermite based Apostol type polynomials ${ }_{H} \mathscr{P}_{n, q, \beta}^{(\alpha)}(x ; k, a, b)$ in terms of the the generalized $q$-Apostol Euler polynomials $E_{n, q, \lambda}(x)$ holds true:

$$
{ }_{H} \mathscr{P}_{n, q, \beta}^{(\alpha)}(x ; k, a, b)=\frac{1}{2} \sum_{m=0}^{n}\left\{\lambda \sum_{r=0}^{n}\left[\begin{array}{l}
n \\
r
\end{array}\right]_{q} E_{n-r, q, \lambda}(x)+E_{n-m, q, \lambda}(x)\right\}{ }_{H} \mathscr{P}_{m, q, \beta}^{(\alpha)}(k, a, b) .
$$

Corollary 3.5. The following explicit relation for the generalized $q$-Hermite based Apostol type polynomials ${ }_{H} \mathscr{P}_{n, q, \beta}^{(\alpha)}(x ; k, a, b)$ in terms of the generalized $q$-Apostol Genocchi polynomials $G_{n, q, \lambda}(x)$ holds true:

$$
\begin{aligned}
H_{n, q, \beta} \mathscr{P}^{(\alpha)}(x ; k, a, b)= & \frac{1}{2[n+1]_{q}}\left\{\lambda \sum_{r=0}^{n+1}\left[\begin{array}{c}
n+1 \\
r
\end{array}\right]_{q} \sum_{m=0}^{r}\left[\begin{array}{c}
r \\
m
\end{array}\right]_{q} G_{n+1-r, q, \lambda}(x)\right. \\
& \left.-\sum_{m=0}^{n+1}\left[\begin{array}{c}
n+1 \\
m
\end{array}\right]_{q} G_{n+1-m, q, \lambda}(x)\right\}_{H} \mathscr{P}_{m, q, \beta}^{(\alpha)}(k, a, b) .
\end{aligned}
$$

In view of Table 1, ceratin explicit representations for the $\operatorname{GqHABP}_{H} B_{n, q, \lambda}^{(\alpha)}(x), \operatorname{GqHAEP}_{H} E_{n, q, \lambda}^{(\alpha)}(x)$ and GqHAGP ${ }_{H} G_{n, q, \lambda}^{(\alpha)}(x)$ are established and are given in Table 3. 
Note. It is to be observed that for $\lambda=1$, the results derived above for the generalized $q$-Hermite-Apostol Bernoulli polynomials ${ }_{H} B_{n, q, \lambda}^{(\alpha)}(x)$, the generalized $q$-Hermite-Apostol Euler polynomials ${ }_{H} E_{n, q, \lambda}^{(\alpha)}(x)$ and generalized $q$-Hermite-Apostol Genocchi polynomials ${ }_{H} G_{n, q, \lambda}^{(\alpha)}(x)$ gives the analogous results for the generalized $q$-Hermite Bernoulli polynomials ${ }_{H} B_{n, q}^{(\alpha)}(x)$, the generalized $q$-Hermite Euler polynomials ${ }_{H} E_{n, q}^{(\alpha)}(x)$ and generalized $q$-Hermite Genocchi polynomials ${ }_{H} G_{n, q}^{(\alpha)}(x)$.

\section{Acknowledgements}

This work has been done under the Senior Research Fellowship (Award letter No. F./2014-15/NFO-2014-15-OBC-UTT24168/(SA-III/Website)) awarded to the second author by the University Grants Commission, Government of India, New Delhi.

\section{References}

[1] G.E. Andrews, R. Askey, R. Roy, Special Functions Encyclopedia of Mathematics and its Applications, Cambridge University Press, Cambridge, 1999.

[2] G.E. Andrews, R. Askey, Classical orthogonal polynomials, C. Brenziniski et al. (editors), in "Polynômes Orthogonaux et Applications", Lecture Notes in Mathematics, Springer-Verlag, Berlin, 1171 1984, pp 36-63.

[3] N. I. Mahmudov, Difference equations of q-Appell polynomials, Appl. Math. Comput., 245 (2014), 539-543.

[4] Q. M. Luo, H. M. Srivastava, Some generalizations of the Apostol-Bernoulli and Apostol-Euler polynomials, J. Math. Anal. Appl., 308 (2005), 290-302.

[5] Q. M. Luo, H. M. Srivastava, Some relationships between the Apostol-Bernoulli and Apostol-Euler polynomials, Comput. Math. Appl., 51(3-4) (2006), 631-642.

[6] Q. M. Luo, Apostol Euler polynomials of higher orders and gaussian hypergeometric functions, Taiwanese J. Math., 10 (2006), 917-925.

[7] Q. M. Luo, H. M. Srivastava, Some generalizations of the Apostol-Genochhi polynomials and the stirling numbers of the second kind, Appl. Math. Comput., 217 (2011), 5702-5728.

[8] T. Ernst, On certain generalized q-Appell polynomial expansions, Ann. Univ. Mariae Curie-Sklodowska Sect. A, 68(2) (2015), 27-50.

[9] M. A. Ozarslan, Unified Apostol-Bernoulli, Euler and Genocchi polynomials, Comput. Math. Appl., 62(6) (2011), 24522462.

[10] B. Kurt, Notes on unified q-Apostol type polynomials, Filomat, 30 (2016), 921-927. 